
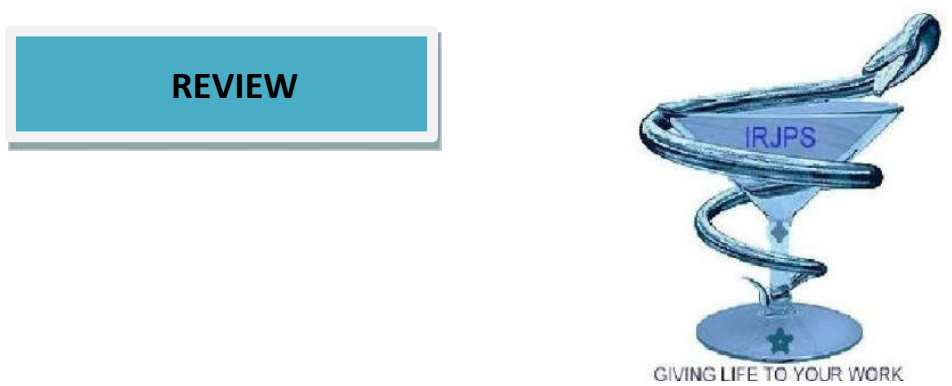

\title{
A REVIEW ON CLEANING VALIDATION FOR PHARMACEUTICAL INDUSTRY
}

\author{
Prashant Tomar*, Preeti Khothiyal \\ Division of Pharmaceutical Sciences, Shri Guru Ram Rai Institute of Technology and Science, Patel Nagar, \\ Dehradun-248001, Uttarakhand, India.
}

\section{Submitted on: 18.05.17; $\quad$ Revised on: 27 .05.17; Accepted on: 31.05.17}

\begin{abstract}
:
The objective of this review is establishment of importance of cleaning validation in pharmaceutical industry. It provides background on cleaning validation in order to prevent the potential problems which may have effect on safety, efficacy and quality of pharmaceutical product being manufactured. Regulatory agencies like US FDA have recommended having an effective cleaning validation program. It briefly provides an overview on cleaning mechanisms, cleaning agents, methods of cleaning, sampling techniques, methods to determine acceptance criteria and analytical techniques to identify and quantify drug residues.
\end{abstract}

KEYWORDS: Cleaning validation, Acceptance Criteria, Maximum Allowable Carryover (MAC), Acceptable Daily Exposure (ADE), Analytical methods.

Corresponding Author: Prashant Tomar, E-mail address: rphtomar@gmail.com
Indian Research Journal of Pharmacy and Science; 13(2017) 950-962

Journal Home Page: https://www.irjps.in

DOI: 10.21276/irjps.2017.4.2.2 


\section{INTRODUCTION}

Validation: The prime goal of any pharmaceutical industry is to manufacture or produce products of required attribute and quality consistently, at the lowest possible cost. Validation studies have been conducted in the pharmaceutical industry of long era still there is an ever-increasing interest in validation because of industry's greater emphasis in recent years on quality assurance program and is fundamental to an efficient production operation. ${ }^{1}$

Validation is a concept that evolved in united states in 1978. The concept of validation has extended through the years to adopt a range of activities from analytical methods used for the quality control and quality assurance of drug substances and drug products for development, production, clinical trials, or process control, Validation is best viewed as a vital and integral part of cGMP. ${ }^{2}$

According to US-FDA Validation is defined as "Process validation is establishing documented evidence which provides a high degree of assurance that a specified process will consistently produce a product meeting its pre-determined specifications and quality characteristics." 3

Thus, Cleaning Validation can be defined as "A documented evidence with high degree of assurance that one can consistently clean a system or piece of equipment to predetermined and acceptable limits."

Cleaning validation is primarily applicable to the cleaning of process manufacturing equipment in pharmaceutical industry. It is necessary to have effective cleaning programs in place because of regulatory requirements. ${ }^{4}$

The focus of cleaning validation is those cleaned surfaces that, if inadequately cleaned, could potentially contaminate the product later manufactured in that same equipment. This primarily covers product contact surfaces in the cleaned equipment. Cleaning validation is not performed only to satisfy regulatory authorities. The safety of patients is the primary goal, and product contamination presents serious liability issues for any pharmaceutical manufacturer or contract organization. $^{5}$

\subsection{Need of Cleaning Validation:}

1. To maintain product integrity: To maintain product integrity basically includes preventing cross-contamination as there might be the possibility that earlier product manufactured in the equipment may get into the product which is going to be manufactured in the same equipment. Contamination may also occur due to equipment residues, drug excipients and cleaning agents. ${ }^{6}$ Another contamination may be due to microbial and/or endotoxins which may lead to safety issues in pharmaceutical dosage forms and may also affect the stability of the product. ${ }^{7}$

2. For equipment reuse: Almost all of manufacturing equipment used in pharmaceutical manufacturing is made of stainless steel which is expensive thus, cannot be disposed of after use and should be reused. Therefore, validated cleaning program should be performed. ${ }^{8}$

3. Due to Regulatory requirements: All the Regulatory agencies recommends for an effective cleaning validation program which includes the FDA, GMPs etc. ${ }^{9}$ The U.S. Food FDA has strict regulations for the cleaning validation which necessarily includes written procedures, equipment design, analytical methods, acceptance criteria etc. $^{10}$

4. Other requirements for cleaning validation may include: Initial qualification of process/ equipment, Critical change in a cleaning procedure, Critical change in formulation, Change in a cleaning process or Change in a cleaning agent. ${ }^{11}$

\section{CLEANING MECHANISMS}

Cleaning is the process of removing unwanted substance or contaminant from the surface of manufacturing. There are several mechanism that remove or aids in removing the contaminants from 
the equipment surfaces. $^{12}$ The various cleaning mechanisms include:

1. Solubility: Solubility here is the dissolution of the contaminant in a liquid media or solvent. For instance some component (salt) might be soluble in water while other in hexane. However the rate of solubility, insoluble form left and solvent used for cleaning is considered in solubility. ${ }^{13}$

2. Solubilization: Solubilization is basically same as solubility the difference is it involves the addition of some substance to pure solvent to make the residue soluble, such as addition of surfactant to the purified water or $\mathrm{pH}$ modifier to make residue in the ionized or unionized hence soluble. ${ }^{14}$

3. Emulsification: This process basically involves the breaking an insoluble liquid residue into tiny droplets and then making those droplets suspended throughout the water or any other specific solvent. ${ }^{15}$

4. Dispersion: Dispersion involves the wetting followed by desegregation and then the formation of suspension of solid particles in water. This mechanism is like emulsification, difference is dispersion being used for cleaning of solid residues. ${ }^{16}$

5. Wetting: This involves the substitution of one fluid from a surface (manufacturing equipment) by another fluid (the cleaning solution). ${ }^{17}$ Wetting of surfaces involves wetting of the soil to be removed as well as wetting of the surface to be clean as the Wetting of the soil to remove provides rapid solubilization, emulsification or dispersion. The main mechanism involved in wetting is the lowering of surface tension making equipment surface easy to clean as well as better penetration of the cleaning solution into cracks and crevices, which are difficult places to clean. ${ }^{18}$
6. Hydrolysis: This involves the cleavage of different bonds in an organic molecule. Hydrolysis is a very effective cleaning procedure because it converts the large water insoluble molecule into smaller watersoluble molecule because the smaller molecule formed is slightly more polar but the resultant hydrolyzed residues might be either water soluble or solubilize at the $\mathrm{pH}$ of the cleaning solution. Thus after the hydrolysis cleaning with water or any other solvent with specific $\mathrm{pH}$ may be used. ${ }^{19}$

7. Oxidation: Oxidation involves the cleavage of various organic bonds by the strong oxidizing agent such as sodium hypochlorite. The oxidants cleaves organic molecule at various linkages in the larger molecule which leads to small molecules that are more polar and increase the water solubility of the degraded components. The effect is similar to that of hydrolysis, except that the phenomenon of oxidation is more universal (and less specific) than hydrolysis. $^{20}$

\section{CLEANING AGENTS}

There is variety of cleaning agents available for cleaning processes. Cleaning agent should be selected based on the Suitability to remove product residues, Compatibility with the equipment, Ease and sensitivity of analytical method, Ease of removal \& verification of removal and most important Toxicity should be low as it may cause harmful effect to cleaning personal or might affect another batch going to be manufactured ${ }^{21}$ Classification of Cleaning Agents:

1. Organic Solvents

2. Aqueous Solvents
i) Water
ii) Surfactants
iii) Chelants
iv) Acids, bases and oxidants. ${ }^{22}$

3.1. Organic Solvents: These can be used as a cleaning agent only if the drug being manufactured is soluble in an organic 
solvent and if organic solvent is also the same solvent used in the bulk active manufacturing process. However safety must be taken in consideration the use of solvents, which may be flammability of the solvent or the occupational exposure limit. Examples of typical organic solvents used are Methanol, Acetone or Ethyl acetate etc. $^{23}$

3.2. Aqueous Solvents: Aqueous cleaning consists of cleaning with water with or with variety of other modified solvents.

i. Water: It is the universal solvent. If water alone is efficient in cleaning the product without much time or physical effort to remove the residues, the water can be best solvent to be used, However the water alone requires too much of time for cleaning. Thus in these conditions the other Solvents come into the picture. ${ }^{24}$

ii. Surfactants: Surfactants used for cleaning generally have a hydrophilic ("water loving") polar end and a lipophilic ("oil loving") non-polar end. The function of a surfactant is for dispersion, emulsification, wetting surfaces and solubilization. As broad categories, surfactants are divided into nonionics, anionic, cationics and amphoterics. Nonionics are mostly used when cleaning mechanism emulsification is required, while anionics are used where wetting and dispersion are required. ${ }^{25}$

iii. Bases: Bases include hydroxides such a s sodium hydroxide or potassium hydroxide. Bases are used to raise the $\mathrm{pH}$, thus rendering certain acid residues more soluble. They also can assist in the hydrolysis of esters or arnides. Finally, bases assist in potentiating surfactants so that the detergency is improved. ${ }^{26}$

iv. Acids: Acids include weak to medium strength acids such as glycolic acid, phosphoric acid, and citric acid. Strong mineral acids such as hydrochloric acid and sulfuric acid are generally not used because of safety reasons or deleterious effects on equipment. Acids are used to lower the $\mathrm{pH}$, thus rendering certain alkaline residues more soluble. ${ }^{27}$

\section{CLEANING METHODS}

Cleaning methods are selected on the basis the extent of disassembly of equipment and on the method of contact of the cleaning agent with the equipment to be cleaned. Cleaning methods are broadly classified into Automated Cleaning and Manual Cleaning. ${ }^{28}$

\subsection{Automated Cleaning:}

i. Clean in Place (CIPs): CIP is an automated process in which disassembly is not performed and in which process equipment is merely flooded with cleaning solution. ${ }^{29}$ Generally a CIP involves spray devices to distribute the cleaning solution to all parts of the equipment. CIP systems are usually designed for cleaning performed with the aid of Aqueous Cleaning agent. ${ }^{30}$ The advantages of CIP includes that they are Designed for better clean ability, Automated, Consistent, Water/cleaner savings, Time savings, Ease of validation and Safe during operation and few disadvantages like Lack of flexibility, High initial capital cost and Uses aggressive cleaning agents. $^{31}$

ii. Agitated Immersion: Agitated immersion is basically filling the cleaning solution into the vessel to be cleaned and then agitating it with the aid of agitator placed already in the vessel, followed by multiple times rinsing with suitable solvent. ${ }^{32}$ The cleaning solution may be an organic solvent or an aqueous solvent due to which cleaning takes place. This process of Agitated Immersion can be performed by using different parameters like various times, temperatures, cleaning agents, and flow conditions. The major advantages of Agitated Immersion are Low Capital cost, Simplicity followed by disadvantages like high process times. ${ }^{33}$ 
iii. Automated Parts Washing: In this process the equipment is disassembled and the equipment parts are placed in a mechanical washer followed by cleaning, rinsing, and drying cycles with the aid of cleaning solution and rinsing solvent which are sprayed to all surfaces of the objects to be cleaned by spray jets and nozzles. ${ }^{34}$ The performance of the automated washer is due to both the mechanical spray systems and due to the chemical action of the cleaner used. The major advantages of Automated Parts Washing are Consistency in performance, Time savings, safe, Chemical and water saving. The disadvantages of automated parts washers are as follows: Initial capital cost and Unsuitable for delicate parts. ${ }^{35}$

iv. Ultrasonic Washers: These cleaning system work on the principle Cavitation in which the sound waves cause tiny bubbles to form when these bubbles grow, they eventually collapse upon themselves. This mechanical energy formed due to collapsing cause dislodge of the residues present on the equipment surface. ${ }^{36,37}$ Ultrasonic washers run at temperatures below about $50^{\circ} \mathrm{C}$. This process is most favorable for cleaning delicate parts that might be damaged by other cleaning processes or parts containing small holes which are difficult to clean. The advantages of ultrasonic are excellent cleaning for delicate items and low initial capital cost but may have the disadvantages like high manual processing and validation issues. ${ }^{38}$

\subsection{Manual Cleaning:}

There are various Process of Manual Cleaning processes which include wiping, high pressure spraying, sink brushing, and equipment brushing. ${ }^{39}$ These all manual cleaning operations has an advantage like low capital costs and disadvantage like higher variability. If done in right way, they are very effective and also allow operators to have high degree of immediate feedback on their cleaning performance being performed. ${ }^{40}$

\section{SAMPLING TECHNIQUES}

The Sampling Techniques must be consistent with sound scientific judgment and must support the goal of the study, which is to prove that the amount of residue or target substance in the equipment has been reduced to an acceptable level. ${ }^{41}$ Equipment is characterized into hot spots and critical sites. Hot spot is the place that is likely to become dirty during the manufacturing process and it is difficult to clean. ${ }^{42}$

There are two generals types of sampling that have been found acceptable which are Direct Sampling technique and Indirect Sampling technique. ${ }^{43}$

\subsection{Direct Sampling Technique: Direct} surface sampling can be further divided into two parts:

i. Visual Inspection (Qualitative): To physically look at and examine the direct surface of the equipment to confirm that contaminants, impurities, residual product, etc are removed. ${ }^{44}$ Visual Inspection employs the following: eyesight of the viewer, available light for viewing, distance of the viewer from the surface, angle of the light and the viewer to the surface, naked eyes (pre-qualification eyes exam is required), boroscope, mirrors, extension poles, bright lighting, white and black cloths. ${ }^{45}$

ii. Swab Sampling (Quantitative): It basically involves the use of Swabbing material, often saturated with a solvent, to physically sample the equipment surfaces. The Swabs are treated with the liquid medium (usually a good solvent for the target substance to ease the absorption of the residue) and then swab is applied on a predetermined surface area and then the swabs are prepared and stored in a suitable container for further analysis. ${ }^{46}$ It is the most preferred technique by most regulatory agencies. Swab material typically consists of polyesters, glass wool, cotton and filter paper. The swab must be compatible 
with the diluent and must give greater flexibility in choosing the proper solvent to check the removal of the targeted substance than swab sticks. ${ }^{47}$ The amount of solvent used to extract the swab should be accurately determined. The choice of the solvent is taken into consideration the solubility of the residue in the solvent, the time needed for the residue to be solubilized, the potential interference/interactions effects between rinsing agents and residue, impact of solvent on the sample surface. ${ }^{48}$

\section{Advantages of Swab Sampling:}

i) Dissolve and physically remove sample.

ii) Adaptability to wide variety of surfaces.

iii) Economically and widely available.

iv) May allow sampling of a defined area.

v) Applicable to active, microbial, and cleaning agent residues. ${ }^{49}$

\section{Limitation:}

i) An Invasive technique that may introduce fibers.

ii) Results might be technique dependent. Swab material and design may inhibit recovery and specificity of the method.

iii) Evaluation of large, complex and hard to reach areas difficult. ${ }^{50}$

\subsection{Indirect Sampling or Rinse Sampling:} Rinse Sampling involves passing a known volume of solvent over a large area and analyzing the recovered solution. ${ }^{51}$ The Rinse sampling is more commonly used in bilk pharmaceuticals Rinse sampling involves using a liquid to cover the surfaces to be sampled. It is one of the easy and widely used sampling methods; most preferable liquid for rinsing is water and rinse volume is an important factor that has to be determined. ${ }^{52}$

\section{Rinse volume $\alpha$ (1/Residue conc. in rinse sample) \\ Limitation:}

i) Limited information about real surface cleanliness in some cases. ii) May lower test sensitivity.

iii) Residues may not be homogenously distributed.

iv) Inability to detect site of residues.

v) Rinse volume is critical to make sure correct interpretation of results. ${ }^{53}$

\section{ACCEPTANCE CRITERIA}

The establishment of meaningful criterion is very important for a cleaning validation program. Acceptance criteria is the foundation stone of the overall program as they give direction as to the effectiveness of cleaning procedure and influence the limits of the analytical method validation. ${ }^{54}$

The FDA does not set specific acceptance criteria for level of residue due to different variety of processes, equipment and products in pharmaceutical country, however FDA quotes that "The firm's rationale for the residue limits establishment should be logical based on the manufacturer's knowledge of the materials involved and be practical, achievable, and verifiable". 55

Thus, Acceptance criteria should be scientifically justifiable, pacifically achievable, and methodically verifiable. $^{56}$

There can be possible 3 types of limits if illustrated below:

1. Visually clean Approach

2. Chemical Approach

3. Microbiological Approach

6.1. Visually clean Approach: GMPs require inspection for visual cleanness before manufacture of any product. Visual inspection is an active observation of the visually accessible product contact surfaces of the pharmaceutical manufacturing equipment

Few key points to be considered in this approach may be angle of view of the inspector, distance from equipment, surface Lighting conditions,

and viewer's knowledge. During inspection viewer can use visual aids which may be limited to additional lighting, magnifying glass, fiberoptic scope or UV light based on the product being inspected and severity. ${ }^{57}$ 
However, there is no such quantifiable limit set by regulatory agencies but most of the industrial experts consider a typical visual limit as not less than $4 \mu \mathrm{g} / \mathrm{cm}^{2}$ and also PIC/S advocates considered to do spiked coupon study for determination of visual limits.

This approach has various advantages like easy to do, can be time and money-saving but it should not be considered for potent drug, microbiological contamination or endotoxin because trace amount of these cause a serious health hazard. ${ }^{58}$

6.2. Chemical residue Approach: ${ }^{59}$ This approach may be based on following criteria's -

i. Therapeutic dose based criteria: Based on the assumption that $1 / 1000$ part of therapeutic dose does not have any clinical impact on human (animal) body and found to be Most suitable for drug product (finished product) manufacturing facility. In this criterion the limit for Maximum Allowable Carryover (MAC) is calculated by the formula given below) of Product $\mathrm{A}$ (Previous) to Product B (Next):

$M A C=\frac{S T D D(\text { Previous }) \times \text { MBS }(\text { Next })}{S F \times S T D D(\text { Next })}$

Where,

STDDprevious - Standard Therapeutic Daily Dose of the investigated product (in the same dosage from as TDDnext) (mg/day) , STDDnext - Standard Therapeutic Daily Dose for the next product (mg/day), MBSnext - Minimum batch size for the next product(s) (mg), SF - Safety factor

ii. Health-based data based criteria: Based on the toxicological information available in Material Safety Data Sheets. Most suitable for active drug (API) manufacturing facility, where cleaning agents are used other than water.
Step 1 - Calculation of the ADE (Acceptable Daily Exposure)

$$
A D E=\frac{N O E L \times \mathrm{BW}}{U F C \times M F \times P K}
$$

Where,

NOAEL - No Observed Adverse Effect Level ( $\mathrm{mg} / \mathrm{kg} /$ day), BW - Is the weight of an average adult in $\mathrm{Kg}$, UFc - Composite Uncertainty Factor: combination of factors which reflects the inter- individual variability, interspecies differences, MF Modifying Factor: a factor to address uncertainties not covered by the other factors and PK - Pharmacokinetic Adjustments

Step 2 - Calculation of MAC from ADE

$$
M A C=\frac{A D E(\text { Previous }) \times \text { MBS (Next) }}{S T D D(\text { Next })}
$$

iii. LD50 based criteria: In cases where no other data is available (e.g. ADE etc) and only LD50 data is available (e.g. chemicals, intermediates, detergents etc), the MAC can be based upon LD50 data.

Step 1 - Calculation of NOEL

$$
N O E L=\frac{L D 50 \times \mathrm{BW}}{2000}
$$

Where,

NOELprevious - No Observed Effect Level (mg/day), LD50 - Lethal Dose 50 in $\mathrm{mg} / \mathrm{kg}$ animal $(\mathrm{mg} / \mathrm{kg})$, BW - Is the weight of an average adult (e.g. $70 \mathrm{~kg})(\mathrm{kg})$ and 2000 is an empirical constant.

Step 2 - Calculation of MAC

$$
M A C=\frac{N O E L(\text { Previous }) \times \text { MBS }(\text { Next })}{S F \times S T D D(\text { Next })}
$$


Safety factors: Safety factors for different dosage forms is given in Table-1

Table I - SAFETY FACTORS FOR DIFFERENT DOSAGE FORMS

\begin{tabular}{|c|c|}
\hline Approach & Approach Typically Applicable To \\
\hline 0.1 to 0.01 & Topical products \\
\hline 0.01 to 0.001 & Oral products \\
\hline 0.001 to 0.0001 & Parenterals, opthalmic products \\
\hline 0.0001 to 0.00001 & Research, investigational products \\
\hline
\end{tabular}

iv. $\quad 10$ PPM criteria: ${ }^{60}$ This criteria is based on the hypothesis that 10 parts of previous product is therapeutically ineffective if presents in million parts of next product, this approach is CGMP requirement and is widely applicable

$M A C=\frac{10 \times \mathrm{BS}}{1000000}$

Where,

BS - Smallest batch size to be available

After the successful calculation of MAC (Maximum Allowable Carryover) the next necessary step is the establishment of the Swab and Rinse limits which are generally used as sampling methods, the establishment of the limit are illustrated below:

6.2.1. Swab Limits: ${ }^{61}$ A recommended value for the Swab can be set; if it is assumed that there is homogenous distribution on all the surfaces. If the total direct contact surface is known, the target value for residue per square meter can be calculated which can be used as vital information for developing a method of analysis and setting up the detection limit. Swab limit can be calculated using the formula:
Target Value $\left(\mathrm{ug} / \mathrm{dm}^{2}\right)$

$$
=\frac{M A C(u g)}{\text { Total Surface }\left({d m^{2}}^{2}\right)}
$$

6.2.2. Rinse Limit: ${ }^{62}$ The residue amount in equipment after cleaning can also be determined by taking rinse samples. The Target value for the Rinse samples can be calculated by formula:

$$
\begin{aligned}
& \operatorname{Target} \operatorname{Value}(\mathrm{mg} / L) \\
& =\frac{M A C(\mathrm{mg})}{\text { Total Surface }(L)}
\end{aligned}
$$

6.3. Microbiological criteria: ${ }^{63}$ This criteria is given in USP Official specifications USP $<1111>$ "Microbial Examination of nonsterile Products: Acceptance criteria for Pharmaceutical Preparations and Substances for Pharmaceutical Use" and is widely used at the Industrial Scale during cleaning validation and it is also an important regulatory requirements.

The various regulatory agencies like European GMP has recommended the microbiological monitoring of the production area during manufacturing of the Pharmaceuticals and generally calculated using the formula: 
cfu/plate $/\left\{\pi \times(5.5 / 2)^{2}\right\} \times$ swab area

\section{ANALYTICAL METHODOLOGY}

When the Acceptance criteria of the target substance is established, the analytical method to test for the targeted substance residues in each swab or rinse sample must be developed or selected which must be consistent with the acceptance criteria. ${ }^{64}$

Regulatory authorities require that the accuracy, limit of Quantitation (LOQ), specificity, linearity and precision of test methods opted for cleaning validation should be established which must be accomplished in accordance with ICH guidelines. These guidelines also applied to the validation of analytical methods that are used to measure residues on the manufacturing equipment. ${ }^{65}$

The basic requirements are:

- The ability to detect the target substance(s) at levels consistent with the acceptance criteria

- The ability to detect the target substance(s) in the presence of other materials that may also be present in the sample (selectivity) ${ }^{66}$

\section{Types of Analytical Method:}

1. Specific methods

2. Non-Specific methods

The selected analytical method must allow qualification of low levels of residue. On the basis of type of Sampling, consideration must be done on how sample preparation may have its influence on method's sensitivity and must relate to the acceptance criteria. $^{67}$

\subsection{Specific Methods:}

i. HPLC: Most preferred method, with this method the Drug residue on Pharmaceutical equipments can be quantified. High Performance Liquid Chromatography (HPLC) make use of injecting sample injection into the separation column, separation of the target substance from other components in the sample, and then measurement of that target substance by the aid of ultraviolet (UV) spectroscopy, conductivity, or evaporative light-scattering detector. ${ }^{68}$

ii. Ultraviolet Spectroscopy: Drug substances in which active chromophoric group is present can be analyzed by this method; it is the most widely available analytical instrument. $^{69}$

iii. Atomic Absorption Spectroscopy: This method is specific to metal ions. Generally used for estimation of sodium or potassium ions during drug residue analysis. ${ }^{70}$

iv. Ion Chromatography: In Analysis of drug residue, this method can be specifically used in determination of anions and cations. It enables the different methods for separation and measurement of anions, which may be present in the detergents or cleaning agents used during cleaning of pharmaceutical manufacturing equipment. ${ }^{71}$

v. Enzyme-Linked Immunosorbent Assay (ELISA): ELISA is mainly used for the determination of protein which if analyzed from any other analytical method might degrade during sample preparation. So, this method is very specific in determination of proteins present in Pharmaceutical formulations. $^{72}$

\subsection{Non - Specific Methods:}

i. Total Organic Carbon: This method uses the oxidation of the substance and measurement of the carbon dioxide generated by using methods such as infrared spectrometry or conductance. The method has disadvantage that it consider that all the measured carbon is due to target substance being analyzed thus making it highly non specific. However this method is generally used in Biotechnological drug residue analysis. $^{73}$

ii. Conductivity: Conductivity measures specifically ions in solution because charge on ions facilitates the conductance of electric current but the conductance will be due all the ions present in the solution, 
which makes this method non specific as unable to detect the nature or type of ion. It can be used for the estimation of an alkaline or an acid cleaning agent. ${ }^{74}$

\section{DISCUSSION}

The Cleaning Validation is an ever-increasing challenge and the topic of interest in the Pharmaceutical industry and is recommended by regulatory agencies like US-FDA, the Cleaning validation demonstrate that manufacturing facilities produce a product which is pure, free from

\section{REFERENCES}

1. Sharma A, Saini S, Process Validation of Solid Dosage Form A Review, International Journal of Research in Pharmacy and Science, 3 (2), 2013, pp. 12- 30.

2. Kaur H, Singh G, Seth N, Pharmaceutical Process Validation: A Review, 3 (4), 2013, pp. 189-194.

3. Potdar MA; Pharmaceutical Quality Assurance. 2nd Edition, Nirali Prakashan, 2009, pp. 6-20.

4. Sharma PP, How to practice GMPs, A Guide for CGMP Compliance along with PAT \& HACCP, 5, 2005, pp. 28-35.

5. Kumar V S, Sanjeev T, Overview of cleaning validation in pharmaceutical manufacturing unit, International Journal of Advanced Research in Pharmaceutical \& Bio sciences, 1(2), 2012, pp. 154164.

6. 21 CFR 210: Current good manufacturing practice in manufacturing, processing, packing, or holding of drugs, 1 April 1997.

7. EPA, Fact sheet: Final air toxics rule for pharrnaceutical production, Washington, D.C, Environmental Protection Agency, Office of Air and Radiation, 1998.

8. Watson D. G, Pharmaceutical Analysis- A Textbook for Pharmaceutical students and Pharmaceutical chemists, $2^{\text {nd }}$ Edition, Edinburgh Elsevier, 2005, Pg.no 11-13.

9. PIC, Recommendations on cleaning validation, document PR1/99-1, Geneva, Switzerland, Pharmaceutical Inspection Convention, 1999. contamination, safe and efficacious in turn controlling the potential carryover of product, intermediates, cleaning agents, microbiological contaminants and extraneous material in subsequent product to the extent which is under acceptable limits. Therefore, the articles covers all the aspects which are required to have an effective cleaning validation program which includes cleaning mechanisms, cleaning agents used, cleaning methods employed, sampling techniques to be used, calculation of acceptance limits and the subsequent analysis of the residues using appropriate analytical method.

10. Food and Drug Administration "Guide to Inspections, Validation of Cleaning Processes" Office of Regulatory Affairs, FDA, Rockville, MD, 1993.

11. Robert Fredrick, the Basic Facts of Cleaning Validation. Canada, Pharmainfo.net, 2004.

12. Rosen M J, Detergency and its modification by surfactants. In Surfactants and interfacial phenomena, New York, John Wiley and Sons Inc, 1978, pp. 272-293.

13. Morgan JJ and Stumm W, Water in Encyclopedia of chemical technology, 4th edition, volume 25, New York, John Wiley and Sons, 1998, pp. 383-388

14. Rosen $\mathrm{M}$ J, Emulsification by surfactants in surfactants and interfacial phenomena, New York, John Wiley and Sons Inc, 1978, pp. 123-148.

15. Rosen M J, Solubilization by solutions of surfactants: Micelles catalysis in Surfactants and interfacial phenomena, New York, John Wiley and Sons Inc, 1978, pp. 224-250.

16. Rosen M J, Dispersion and aggregation of solids in liquid media in Surfactants and interfacial phenomena, New York, John Wiley and Sons Inc, 1978, pp. 251-271

17. Rosen M J, Wetting and its modification by surfactant in Surfactants and interfacial phenomena, New York, John Wiley and Sons Inc, 1978, pp. 174-199.

18. Rosen M J, Reduction of surface and interfacial tension of surfactants in Surfactants and interfacial phenomena, New York, John Wiley and Sons Inc, 1978, pp. 149-173. 
19. Tau K D, V Elango and J A McDonough, Organic in Encyclopedia of chemical technology, $4^{\text {th }}$ edition, volume 9, New York, John Wiley and Sons, 1994, pp.783-786.

20. Kumar S, A review on concept of cleaning validation in pharmaceutical Industry, International Research Journal of Pharmaceutical Sciences, 3(7), 2012, pp. 17-19.

21. Michael $P$, Cleaning validation \& Regulatory compliance an introduction and overview, Merck milipore, pp. 11-18.

URL:http://www.dcvmn.org/IMG/pdf/cleaning_vali dation_michael_payne_pdf

22. LeBlanc, D. A., D. D. Danforth, and J. M. Smith, Cleaning technology for pharmaceutical manufacturing. Pharmaceutical Technology, 1993, 17 (7): 84-92.

23. Health product and food branch inspectorate, Cleaning validation guidelines, 2008, pp. 1-16.

24. Lynn J L, Detergency in Encyclopedia of chemical technology, 4th edition, volume 7, New York, John Wiley and Sons, 1997, pp. 1073-1081.

25. Lynn J L and B H Bory, Surfactant in Encyclopedia of chemical technology, $4^{\text {th }}$ edition, volume 23 , New York, John Wiley and Sons, 1997, pp. 478541.

26. Ghosh A and Dey S, Overview of Cleaning Validation in Pharmaceutical industry, International journal of Pharmaceutical Quality Assurance, 2010, 2(2), pp. 26-30.

27. Patel Payal $\mathrm{K}$ et al, An Overview on Cleaning Validation, International Journal of Pharmaceutical \& Biological Archives, 2011, 2(5), 1332-1336, ISSN 0976 - 3333.

28. Adams D G and Aggarwal D, CIP system design and installation, Pharmaceutical Engineering 10(6), 1990, pp. 915.

29. Stewart J C and D A Seiberling, Clean in place Chemical Engineering, 1996, 103 (1), pp.72-79.

30. Seiberling $\mathrm{D}$ A, Alternatives to conventional process/CIP design for improved cleanability. Pharrnaceutical Engineering, 1992, 12 (12), pp. 1626.

31. PDA Biotechnology Cleaning Validation Subcommitte, Cleaning and cleaning validation A biotechnology perspective, Bethesda, Parenteral Drug Association, USA, 1996, pp. 65-84.
32. Duchi $\mathrm{P} \mathrm{J}$ et al, Cleaning PCBs in Electronics understanding today's needs, Inventac Performance Chemicals, Bry sur Marne, France.

33. Quality Assurance in Industrial parts Cleaning Basics Chemistry and Processes, 2013, SurTec Deutschland, pp. 32-35.

34. Best Technology, Precision Cleaning, Passivation \& Finishing Systems.

URL:

http://www.besttechnologyinc.com/industria 1-parts-washers-cleaning-systems/part-

cleaning-guide/

35. Varghese G and P Lopolito, Cleaning Engineering and Equipment Design, Cleaning and Cleaning Validation, Volume 1, PDA/DHI, Bethesda, Maryland, 2009, pp. 126-127 and 141-142.

36. Azar and Lawrence, Cavitation in ultrasonic cleaning and cell disruption, Controlled Environments, 2009.

URL: http://www.absotecthailand.com/Cavitation.pdf.

37. Williams and Douglas, Guide to Cleaner Technologies: Cleaning and Degreasing Process Changes, Washington DC, United States Environmental Protection Agency, 1994, pp. 19.

38. Fuchs $\mathrm{F}$ J, the key to Ultrasonics-Cavitation and implosion Precision Cleaning, 1995, 3(10), pp. 1317.

39. Anindya G, Sanjay D, Overview of Cleaning Validation in Pharmaceutical Industry, International Journal of Pharmaceutical Quality Assurance, 2010, 2(2), pp. 26-30.

40. Imtiaz HS, Validation standard operating procedures: a step-by-step guide for achieving compliance in the pharmaceutical, medical device and biotech industries, 2006, pp. 1045-1060.

41. Sandeep K, why the Swab Matters in Cleaning Validation Controlled Environments, Health Canada guideline, 2010.

URL: www.ngenetics.com/file/Controlled_Environments_2010.p df

42. Fourman G L and Mullen M V, Determining cleaning validation acceptance limits for pharmaceutical manufacturing operations, Pharmaceutical Technology, 1993, 17 (4), pp. 5460 . 
43. Agallaco J. Frederick Carelton J, A Text Book of Validation of Pharmaceutical Process, Spring Publisher, 3rd Edition, 2008, pp. 525-565.

44. Forsyth R J et al, Visible Residue limit for Cleaning Validation and its Potential Application in Pharmaceutical Research facility, Pharm Technology, 2005, 28(10), pp. 58-72.

45. LeBlanc D A, Visually Clean as solo acceptance criteria for cleaning validation protocols, Journal of Pharmaceutical Science and Technology, 2002, 56(1), pp. 31-36.

46. Lombardo $\mathrm{S}$ et al, Development of surface swabbing procedures for a cleaning validation program in a biopharmaceutical manufacturing facility, Biotechnology and Bioengineering, 1995, pp. 513-519.

47. Cooper D W, using swabs for cleaning validation: A review in cleaning validation, Institute for Validation Technology, USA, 1997, pp. 74-89.

48. Bodavula Samba Siva Rao, cleaning validation of albendazole tablet $400 \mathrm{mg}$, Pharma Innovations, 2012, 1(4), pp. 76-9.

49. Patel Hiren, Popat bhai et al, Process validation of Benzepril HCL $5 \mathrm{mg}$ tablet, International Research Journal of Pharmaceutical and applied sciences, 2012, (4), pp. 1-16.

50. Lakshmana $\mathrm{P}$, et al, Cleaning validation and its importance in Pharmaceutical Industry, Pharma Times, 2010, 42(07), 21-25.

51. Bodavula Samba Siva Rao, Cleaning validation of albendazole tablet $400 \mathrm{mg}$, Pharma Innovations, 2012, 1(4), pp. 76-9.

52. Babita Lodhi et al, Cleaning validation for the pharmaceuticals, biopharmaceuticals, cosmetic and neutraceuticals industries, Journal of Innovations in Pharmaceuticals and Biological Sciences, 2014, Volume-1, (1), 2014, pp. 27-38.

53. LeBlanc D A, Rinse sampling for cleaning validation studies, Pharmaceutical Technology, 22 (5), 1998, pp. 66-74.

54. Recommendation on VMP, IQ and OQ, non-sterile process validation and cleaning validation, Pharmaceutical Inspection Convention, Pharmaceutical Inspection Co-Operation Scheme, 2007.

URL:

https://www.picscheme.org/layout/document.php?id $=152$.
55. FDA, Inspection guide: Cleaning validation. Rockville, Md., USA: Food and Drug Administration, 1992.

56. GMP guide for API", (ICH, Q7). ICH Good Manufacturing Practice Guideline for Active Pharmaceutical Ingredients, July 23 1999, on Cleaning Validation, Health Canada

57. S.W. Harder, The Validation of Cleaning Procedures, Pharm. Technol. 1984, 8 (5), pp. 29-34.

58. Fourman G and Mullin M, Determining Cleaning Validation Acceptance Limits for Pharmaceutical Manufacturing Operations, Pharmaceutical Technology, 1993, pp. 54-60

59. Mendenhall D, Cleaning Validation, Drug Development and Industrial Pharmacy, 1989, 15(13), pp. 2105-2114.

60. Active Pharmaceutical Ingredient Committee (APIC) group of EEFIC, 1999, Guide to cleaning validation in API plants. APIC Publications, pp. 223.

61. Justification of limit for cleaning validation in the manufacture of active pharmaceutical ingredient, 2007, GMP news.

62. Mc Arthur, Vasilevsky, Cleaning validation for biological products case study, pharmaceutical engineering, 1995.

63. USP $<1111>$ "Microbial Examination of nonsterile Products: Acceptance criteria for Pharmaceutical Preparations and Substances for Pharmaceutical Use".

64. Guidelines for Single-Laboratory Validation of Analytical Methods for Trace-Level Concentrations of Organic Chemicals Special Publication - Royal Society of Chemistry: Principles and Practices of Method Validation, 2000, 256, pp. 179- 252.

65. Irsch R B, Validation of Analytical Methods Used in Pharmaceutical Cleaning Assessment and Validation, Pharmaceutical Technology (Supplement), 1998, pp. 40-46.

66. Shifflet $M$ and Shapiro M, Development of Analytical methods to accurately and precisely determine residual Active pharmaceutical

ingredients and cleaning agents on Pharmaceutical Surfaces, Am Pharm, 2002, pp. 35-39.

67. Kumar nitin et al., Development and Validation of a Simple and Rapid Reversed Phase Liquid 
Chromatography Method for Estimation of Pregabalin from Equipment Surfaces Used for Pharmaceutical Manufacturing, Ann Chromatogr Sep Tech, 2016, 2(3), pp. 1023.

68. Goti pp et al, Development and Validation of Analytical Method for estimation of Declofenac Sodium in Swab samples, International Journal of Pharmaceutical Sciences and Research, Volume, 2013, 4(2), pp. 741-744.

69. Sofia Ahmed, Nafeesa Mustaan, et al, Validation of a UV Spectrometric Method for the Assay of Tolfenamic Acid in Organic Solvents, Journal of Pharmaceutics, 2015.

70. Jawla Sunil, Jain Sandeep, Atomic Absorption Spectrometric Method for Estimation of Diclofenac sodium and Mefenamic acid in Pharmaceutical Formulations, International Journal of
Pharmaceutical Sciences and Drug Research, 2010, 2(1), pp. 45-47.

71. Dennis Jenke, Application of Ion Chromatography in Pharmaceutical and Drug Analysis, volume 49, Journal of Chromatographic Science, 2010.

72. Samarajeewa U et.al, Application of immunoassay in the food industry. Critical Reviews in Food Science and Nutrition, 1991, pp, 403-434.

73. Jenkins K M., et al., Application of Total Organic Carbon Analysis to Cleaning Validation," PDA J. Pharmaceutical Sciences \& Technology, 1996, 50 (1), pp. 6-15.

74. Zeyad G. Yasseen, On the Interactions of Bovine Serum Albumin with Some Surfactants: New Insights from conductivity Studies, Journal of Chemical and Pharmaceutical Research, 2012, 4(7), pp. 3361-3367. 